\title{
Simple quasi-analytical holonomic homogenization model for the non-linear analysis of in-plane loaded masonry panels: Part 2, structural implementation and validation
}

\author{
G. Milani ${ }^{*}$, E. Bertolesi ${ }^{1}$ \\ 1 Department of Architecture, Built Environment and Construction Engineering ABC \\ Politecnico di Milano, Piazza Leonardo da Vinci 32, 20133 Milan, Italy \\ *corresponding author e-mail: gabriele.milani@polimi.it
}

\begin{abstract}
The simple quasi analytical holonomic homogenization approach for the non-linear analysis of in-plane loaded masonry presented in Part 1 is here implemented at a structural level and validated. For such implementation, a Rigid Body and Spring Mass model (RBSM) is adopted, relying into a numerical modelling constituted by rigid elements interconnected by homogenized inelastic normal and shear springs placed at the interfaces between adjoining elements. Such approach is also known as HRBSM. The inherit advantage is that it is not necessary to solve a homogenization problem at each load step in each Gauss point, and a direct implementation into a commercial software by means of an external user supplied subroutine is straightforward.

In order to have an insight into the capabilities of the present approach to reasonably reproduce masonry behavior at a structural level, non-linear static analyses are conducted on a shear wall, for which experimental and numerical data are available in the technical literature. Quite accurate results are obtained with a very limited computational effort.
\end{abstract}

Keywords: Masonry; in-plane loads; Rigid Body and Spring Model RBSM; homogenization; commercial code implementation.

\section{INTRODUCTION}

In the first Part of this paper, a simplified homogenization two-step model was proposed for the non-linear structural analysis of masonry walls in-plane loaded [1]-[3]. The first step was discussed in detail. It relies into a mechanical model application at the meso-scale, where a regular assemblage of bricks and mortar forming a Representative Element of Volume REV was substituted by means of a macroscopic equivalent material through a so called compatible identification procedure. The unit cell was roughly meshed by means of 24 triangular elastic plane stress elements (bricks) and interfaces (mortar). For interfaces, both a piecewise linear and an exponential law formally identical to an improved version of the Xu-Needleman law were implemented is a non-commercial code. The limited joints tensile strength and the post-peak softening in both tension and compression, eventually characterized by a coupling between normal and shear stresses were suitable accounted for. The procedure allows for a consistent characterization of the macroscopic masonry behavior by means of a rigorous procedure that suitably reproduce the orthotropy along material axes, softening in tension and compression and the frictional behavior under shear and coupled shear/compression stress states. Here the second step is discussed in details, which relies into the structural implementation of the homogenized in-plane stress-strain behavior found in the previous step. The procedure allows a realistic analysis of entire masonry walls in-plane loaded in the non-linear static range, without the need of adopting cumbersome distinct discretizations of blocks and mortar at a structural level (micro-mechanical approach) or the utilization of macroscopic models where the characterization of the mechanical properties requires costly experimental campaigns and should be repeated case by case. The structural implementation is obtained meshing entire walls through rigid infinitely resistant quadrilateral elements [4]-[6] interconnected by non-linear interfaces. Mechanical properties of the interfaces are obtained by means of the aforementioned homogenization procedure. From a mechanistic point of view, each interface between adjoining elements can be thought as constituted by two normal springs placed at the corners and a shear spring interconnecting opposite nodes of the interface. The discretization is typically inspired by that proposed by Kawai [4] for isotropic non linear continua, but rigorously accounts for the different strength exhibited by masonry along material axes both in the linear and non-linear range and the quite marked softening behavior. The structural implementation is entirely handled within the commercial software Abaqus [7]. The implementation into a robust commercial code secures robustness of the algorithm when dealing with non-linear static analyses, especially in the softening branch and in presence of many finite elements, as well as a straightforward generalization of the model to the non linear dynamic range.

A benchmark on a shear wall tested experimentally at ETH Zurich some decades ago [8] is reported to have an insight into the capabilities of the procedure proposed, as well as comparisons with the outcome provided by a previously presented macroscopic model [9] are also discussed in detail. 


\section{THE NUMERICAL MODEL}

The homogenized mechanical properties deduced using the model presented in Part 1 are implemented on a commercial FE code to simulate the behavior of entire panels up to failure. The aim is to show that the present models can be adopted by practitioners for the analysis of large scale structures, for which the classical micromechanical approach requires unpractical computational cost, whereas common commercial codes have implemented only isotropic macromodels. The simulations, carried out using Abaqus [7], deal with a variety of masonry panels subjected to different load conditions for which experimental data are available in literature, as well as numerical results provided by other authors. For the sake of conciseness, here only one example is reported and the reader is referred to [10] for a comprehensive discussion on other benchmarks. The homogenized stress-strain relationship to be used at structural level requires an identification of the spring elastic properties, in order to make the rigid-spring assemblage model compatible with the orthotropic continuum. Classically (see Kawai [4] for the general framework and recent applications for masonry by Casolo \& Milani [5][6]) such match is achieved in the linear elastic range by energy equivalence. Let us consider two rectangular rigid elements linked with a homogenized interface, having geometric properties as in FIGURE 1. Let us denote the dimensions of the rigid elements with " $\mathrm{L}$ " and " $\mathrm{H}$ ", respectively for the length and the height, and the out of plane thickness with " $t$ ". The thickness of the interfaces is identified with "th". Under the application to the mechanical system of a normal displacement $\delta$, the strain of the homogenized material is $\varepsilon=\delta /(2 L+t h)$, whereas the deformation of the springs is $\widetilde{\varepsilon}=\delta / t h$. The volumetric strain energies of the homogenized and rigid-spring models are:

$$
\begin{aligned}
& U_{\mathrm{xx}}=V \cdot \frac{1}{2} \cdot \sigma \cdot \varepsilon=\frac{1}{2} \cdot \frac{\delta^{2}}{2 L+t h} \cdot \tilde{E}_{x x} \cdot H \cdot t \\
& U_{\mathrm{xx}}=V \cdot \frac{1}{2} \cdot \sigma \cdot \varepsilon=\frac{1}{2} \cdot \frac{\delta^{2}}{t h / 2} \cdot E_{n x x} \cdot H \cdot t
\end{aligned}
$$

Where $\tilde{E}_{x x}$ is masonry homogenized elastic modulus along the horizontal direction, $E_{n x x}$ is the elastic modulus of normal springs within the rigid elements and spring mass structural model and $V$ is the volume of the mechanical system in FIGURE 1-b.

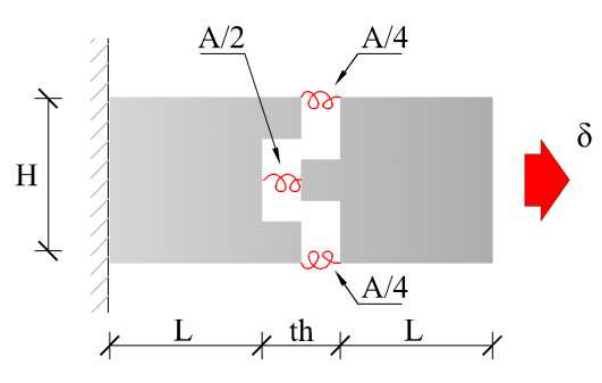

a

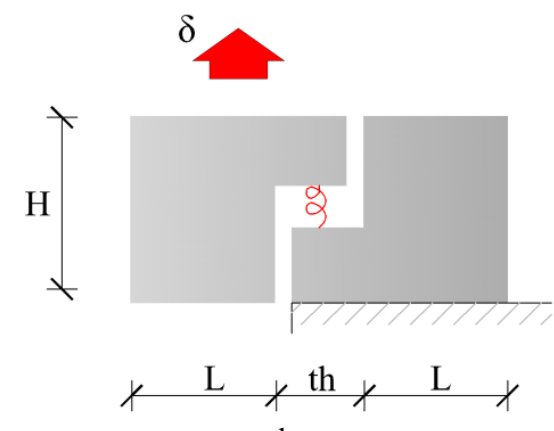

b

Equating the two energies, we obtain the elastic modulus of the axial (horizontal normal) springs as:

$\mathrm{E}_{\mathrm{nxx}}=\frac{t h / 2 \cdot E_{x x}}{2 L+t h}$

Using the same procedure it is possible to define Enyy for vertical normal springs as:

$\mathrm{E}_{\mathrm{nyy}}=\frac{t h / 2 \cdot E_{y y}}{H+t h}$

The determination of $\mathrm{G}_{\mathrm{nxy}}$ follows an analogous procedure, see FIGURE 1-b, allowing an estimation of Gxy as follows.

$U_{\mathrm{xy}}=\frac{1}{2} \cdot \frac{\delta^{2}}{H / 2} \cdot G_{n x y} \cdot t h \cdot t$

$U_{\mathrm{xy}}=\frac{1}{2} \cdot V \cdot \delta^{2} \cdot G_{n x y}=\frac{1}{2} \cdot \frac{\delta^{2}}{2 L+t h} \cdot H \cdot t$

$\mathrm{G}_{\mathrm{nxy}}=G_{x y} \cdot \frac{H^{2} / 4}{t h \cdot(2 L+t h)}$ 


\section{RESULTS}

The simulations presented refer to a masonry shear panel tested by Ganz and Thürlimann [8] at ETH Zurich some decades ago. The experimental campaign, which is now commonly used to benchmark new numerical models in the field of masonry modeling, was carried out with the aim of better understanding the structural behavior of three geometrically identical masonry panels under the combined action of different vertical pre-compressions and shear. Three different series of shear panels with almost the same geometry but under different loading conditions, labeled as W1, W2 and W3 were tested. Here, only shear panel W2 results are taken into consideration for the sake of conciseness. The walls have in-plane dimensions equal to $3600 \times 2000 \mathrm{~mm}^{2}$ (width $\mathrm{x}$ height), see FIGURE 2 . Hollow clay bricks of dimensions equal to $300 \times 200 \times 150 \mathrm{~mm}^{3}$ were used, whereas the thickness of the joints is assumed equal to $10 \mathrm{~mm}$. Two stiff masonry flanges are present in correspondence of vertical edges and a thick heavy concrete plate was put on the top, to properly apply and distribute the desired vertical pre-compression load, variable depending on the sample tested, FIGURE 2-a. WP1 and WP2 are subjected, prior the application of the horizontal load, to a distributed vertical precompression equal respectively to 0.61 and $1.91 \mathrm{MPa}$, whereas a concentrated eccentric load equal to $422 \mathrm{kN}$ is applied on the top edge of panel WP3. The width of the flanges is equal to a single brick and their out-of-plane thickness is equal to $600 \mathrm{~mm}$ for WP1 and WP2 and $840 \mathrm{~mm}$ for WP3. The utilization of hollow bricks makes convenient the utilization at a structural level of the homogenized stress strain relationships depicted in FIGURE 3, appearing reasonably in agreement with those used in the technical literature to numerically reproduce the present experimental studies. Experimental evidences shown that during the first phase of application of the horizontal imposed displacement, diagonal cracks started to occur with a typical stepped pattern, while at the end of the tests the collapse mechanism is characterized by the formation of flexural hinges on both flanges. All panels, but especially WP2 which has a high pre-compression level, exhibited a global experimental ductile behavior, see FIGURE 2-b where experimental curves and numerical results are represented. The panel is discretized by means of a fairly refined mesh constituted by 210 rigid elements (dimensions equal to 150x190 mm2), FIGURE 4.

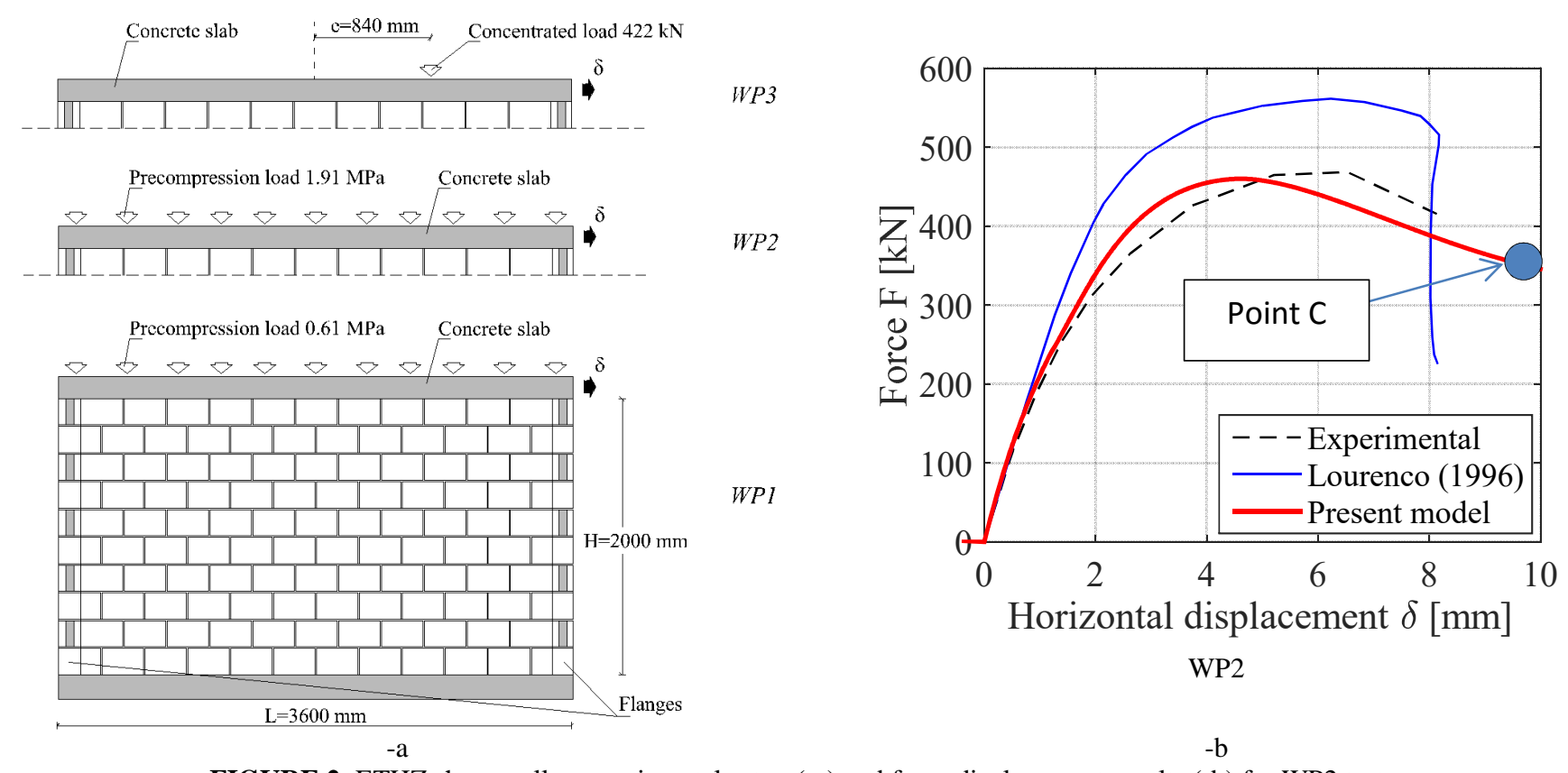

FIGURE 2: ETHZ shear walls, experimental setup (-a) and force displacement results (-b) for WP2 test

As already pointed out, in FIGURE 2-b global force-displacement curves obtained numerically are compared with experimental ones and those provided by Lourenço [9] with a macroscopic orthotropic elasto-plastic model. As can be noted, rather satisfactory agreement is found. Even the extremely ductile behavior of the specimens seems well reproduced by the proposed approach.

Deformed shapes at the end of the numerical simulations (point $\mathrm{C}$ in FIGURE 2-b) and resultant crack patterns are summarized in FIGURE 3. The deformed shape of the panel at the end of the simulations exhibits some common features with experimented ones, as for instance the formation of a well-defined plastic hinge near the base of the right flange and localization of crushing, i.e. compression failure, in the lower right part of the shear wall, plus the formation of a diagonal strut. As a matter of fact, even if the typical stepped cracks experienced during the loading history cannot be reproduced by any homogenization approach, the deformed shape obtained at the end of the simulations clearly shows the formation of a shear band running on the diagonal compressed strut. 
A visible rotation of the concrete slab is also worth noting, with the formation of a clear region undergoing tensile failure, spreading considerably thanks to the combined effect of pre-compression and concrete slab rotation. The compressed toe on the right, indeed, exhibits high stress values, compensated by the tensile stress region spreading. A mixed shear-crushing failure is also clearly visible near the lower right flange, showing several similarities with experimental evidences.

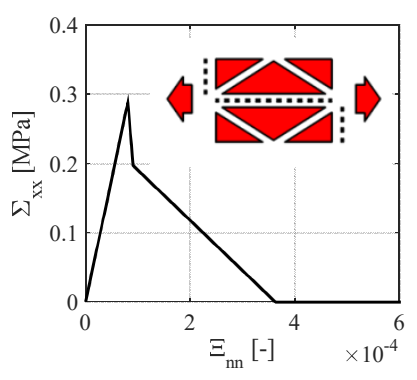

$-a$

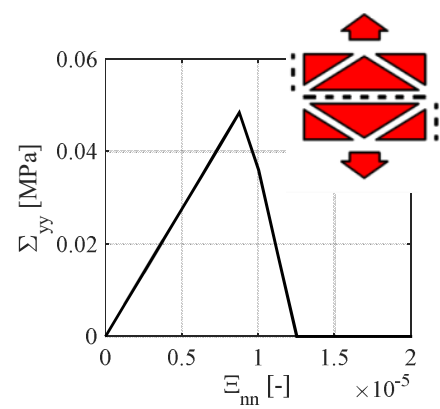

-b

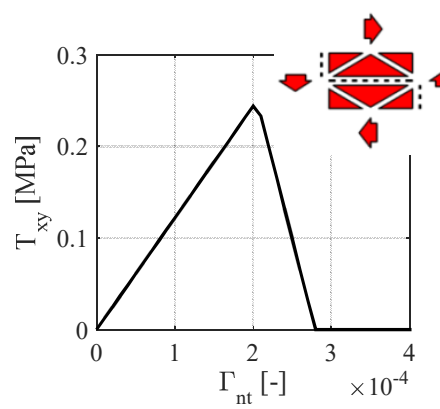

$-c$

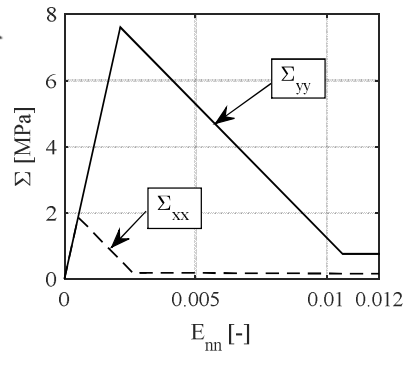

-d

FIGURE 3: ETHZ shear walls, homogenized stress strain relationships adopted at a structural level. -a: horizontal tensile stress behavior. b: vertical tensile stress behavior. $-\mathrm{c}$ : shear stress behavior. $-\mathrm{d}$ : compression behavior.

Point $\mathrm{C}$ damage compression

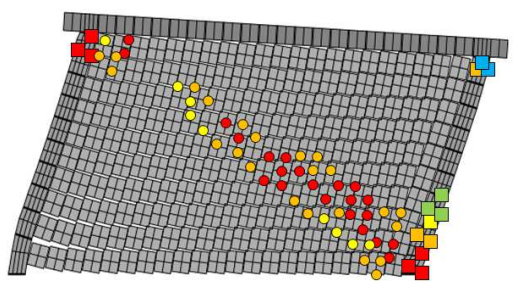

Point $\mathrm{C}$ tension

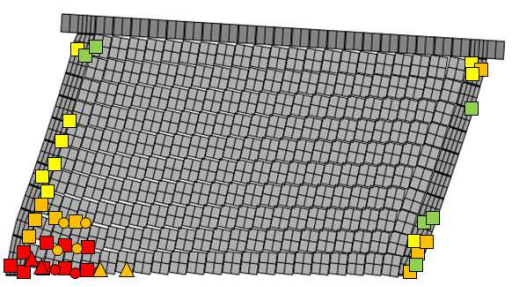

Shear failure

Axial failure $x x$

Axial failure yy

Compressive behavior

Tensile behavior

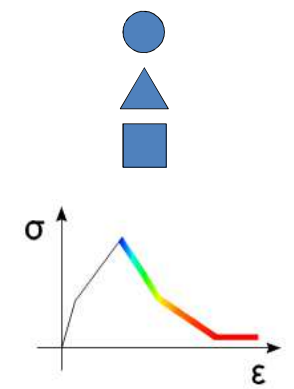

FIGURE 3: ETHZ shear panel WP2, deformed shapes at progressive imposed horizontal deformation and corresponding damage patterns at the end of the simulations.

\section{REFERENCES}

[1] Milani G. Four approaches to determine masonry strength domain. Proceedings of ICE, Engineering and Computational Mechanics 2015; 168(3): 99-121.

[2] Milani G. Simple homogenization model for the non-linear analysis of in-plane loaded masonry walls. Computers and Structures 2011; 89: 1586-1601.

[3] Milani G, Bertolesi E. Holonomic homogenized approach for the non-linear analysis of in-plane loaded masonry panels. Under review 2016.

[4] Kawai T. New discrete models and their application to seismic response analysis of structures. Nucl. Eng. Des. 1978; 48: 207-229.

[5] Casolo S, Milani G. A simplified homogenization-discrete element model for the non-linear static analysis of masonry walls out-of-plane loaded. Engineering Structures 2010; 32: 2352-2366.

[6] Casolo S, Milani G. Simplified out-of-plane modeling of three-leaf masonry walls accounting for the material texture. Construction \& Building Materials 2013; 40: 330-351.

[7] Abaqus User's Manual 2006.

[8] Ganz HR, Thürlimann, B. Tests on masonry walls under normal and shear loading (in German). Report No. $7502-$ 4, Institute of Structural Engineering, ETH Zurich, Zurich, Switzerland, 1984.

[9] Lourenço PB. Computational strategies for masonry structures. PhD Thesis. TU Delft, The Netherlands; 1996.

[10] Bertolesi E. Simple compatible homogenization strategies. Application to unreinforced masonry structures in and out of plane loaded. PhD Thesis, Department ABC, Technical University of Milan, Italy, 2016 (in preparation). 